\title{
Acute urinary retention due to a large isthmic leiomyoma - a case report and literature review
}

\author{
Nicolae BACALBASA ${ }^{1,2}$, Irina BALESCU ${ }^{3}$, \\ Mihaela VILCU ${ }^{1,2}$, Simona DIMA ${ }^{4}$, Iulian BREZEAN ${ }^{1,2}$ \\ 1 "Carol Davila" University of Medicine and Pharmacy, Bucharest, Romania \\ 2 "Ion Cantacuzino" Clinical Hospital, Bucharest, Romania \\ ${ }^{3}$ Ponderas Academic Hospital, Bucharest, Romania \\ ${ }^{4}$ Fundeni Clinical Institute, Bucharest, Romania
}

\begin{abstract}
Uterine leiomyoma represent a common finding in women at fertile age, being usually responsible for the apparition of metrorhagia and pelvic pain; in certain cases presenting large uterine fibroids, tumoral masses can be encountered at the clinical abdominal exam. However, in certain cases they can be responsible for the apparition of urinary signs such dysuria and pollakiuria due to their compression on the urinary bladder. The aim of this paper is to report the case of a 56-year-old patient who presented for oligo-anuria and bilateral ureteral hydronephrosis and who was diagnosed with a large intra-istmic leiomyoma with vaginal development which induced the development of acute urinary retention. The patient was submitted to surgery, a total hysterectomy with bilateral adnexectomy being performed.
\end{abstract}

Keywords: acute urinary retention, uterine leiomyoma, intra-isthmic development

\section{INTRODUCTION}

Uterine fibroids represent a common finding in women at fertile age, being usually diagnosed due to the presence of metrorhagia, diffuse pelvic pain, urinary incontinence or dysuria in cases presenting urinary bladder compression (1). However, acute urinary retention with secondary uretero-hydronephrosis due to uterine fibroids is a rare situation, few cases being reported so far (1-4).

\section{MATERIAL AND METHODS}

The 56-year-old patient with no significant medical history presented herself for diffuse pelvic pain, dysuria for the last 2 weeks in association with oligo-anuria in the last 24 hours.

\section{RESULTS}

The abdominal ultrasound revealed the presence of indirect signs of acute urinary retention bilateral uretero-hydronephrosis and a large tumoral mass developed at the level of the uterine isthmus compressing the urinary bladder and the urethra. The vaginal ultrasound confirmed the presence of the tumoral mass at the level of the uterine isthmus with characteristics suggesting the fibroid origin of the tumor. The patient was submitted to surgery, a total hysterectomy with bilateral adnexectomy being performed. Intraoperatively the cause of the acute urinary retention was proved to be the $5 / 4 / 4 \mathrm{~cm}$ intra-isthmic uterine fibroid, while the histopathological studies confirmed the absence of any malignant elements. 
The postoperative course was uneventful, the patient being discharged in the sixth postoperative day. At one month follow-up the performed ultrasound revealed the absence of residual ureterohydronephrosis, while the patient declared the resolution of the urinary symptoms.

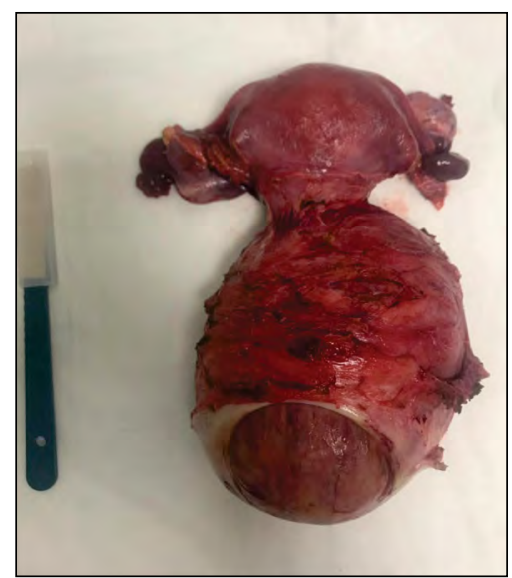

FIGURE 1. The specimen of total hysterectomy with bilateral adnexectomy for a large isthmic uterine fibroid

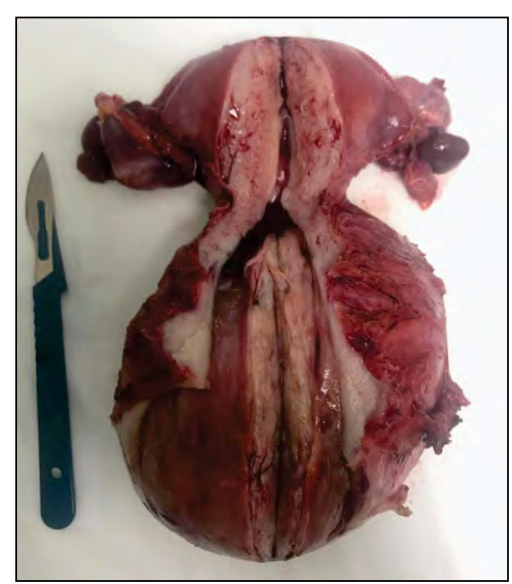

FIGURE 2. The sectioned specimen revealing the fibroid

\section{DISCUSSIONS}

Acute urinary retention is rarely encountered in women, the most commonly encountered causes being related to the presence of pregnancy, the enlarged uterus compressing the ureters, to the association of lumbar disc herniation or other neurological disorders, perineal abscesses, ectopic pregnancy or postoperatively after pelvic surgery (5-7).

However, fibroids induced acute urinary retention is a very rare condition, which, if not treated with radical intent, might induce the apparition of urinary bladder diverticula, vesico-uereteral reflux or recurrent urinary tract infection (4). When it comes to the mechanism of apparition of urinary retention, it seems that the most commonly in- criminated is the mechanical one, consisting of the mechanical compression between the uterine cervix and the symphisis pubis of the bladder neck (8). However, any other condition which might lead to the uterine impactation in the bony pelvis might induce acute urinary retention (9). Other incriminated mechanisms are related to pelvic congestion due to hormonal modifications during menstruation, detrusor ischemia due to fibroid compression or nervous compression due to fibroid compression $(10,11)$.

Moreover, certain authors reported the presence of intermittent urinary retention which is induced by maintaining the patient in the supine position; in such cases patients are able to void while standing but not when sitting or in the supine position due to the compression of the bladder neck and internal urethral orifice (3); in such cases patients initially develop changing of the voiding position followed by intermittent urinary retention, and finally complete acute urinary retention.

When it comes to the therapeutic strategies in such cases, they might consist of various therapeutic approaches ranging from uterine artery embolization to total hysterectomy $(1,4)$.

An interesting series of patients presenting acute urinary retention due to uterine fibroids was published by Singh et al. and included four such cases. Patients' ages at the time of developing acute urinary retention ranged between 25 and 42 years, while the number of fibroids compressing the urinary bladder ranged between one and two, with the largest diameter ranging between $9.3 \mathrm{~cm}$ and $14.2 \mathrm{~cm}$. In all cases patients were submitted to surgery for acute urinary retention and consisted of inter-adnexal total hysterectomy in two cases (with ages of 36 and 39 years), total hysterectomy with bilateral adnexectomy in one case (in the 42-year-old patient) and myomectomy (in the case of the 25-year-old patient) (11).

A review study conducted on this theme and published by Wu et al. included 16 case reports and five case series totalizing 37 cases of patients diagnosed with acute urinary retention due to uterine fibroids. Patients' ages ranged between 27 and 75 years while the dominant dimensions of the uterine fibroids ranged between 5.7 and 22.4 $\mathrm{cm}$ (12). Among the 16 cases included in this study, there were also six cases submitted to surgery for acute urinary retention due to uterine fibroids, by the authors at St. Michael's Hospital in Toronto, Canada from January 2011 to December 2013. All cases included in the study were premenopausal or menopausal and presented the largest diame- 
ter ranging between $7.8 \mathrm{~cm}$ and $13.6 \mathrm{~cm}$; as for the location of the fibroid inducing acute urinary retention, it was located on the posterior uterine surface in two cases, on the anterior surface in one case, a fundic myoma in one case and a central myoma in the last case. As for the type of treatment, it consisted of vaginal hysterectomy in two cases, abdominal hysterectomy in one case, laparoscopic assisted vaginal hysterectomy in one cases, laparoscopic abdominal hysterectomy in one case and ulipristat acetate in one case (12). As for the 31 remnant patients included in the review study, the age at the time of acute urinary retention ranged between 25 and 53 years, most cases presenting posterior myomas. As for the type of treatment, surgery was the option of choice in 24 cases and consisted of laparoscopic or open inter-adnexal hysterectomy or total hysterectomy with bilateral adnexectomy in 20 cases and myomectomy in the remaining four cases, the other seven cases being treated by conservative therapies including uterine artery embolization. However, in all cases urinary symptoms disappeared once the cause was solved (11).

\section{CONCLUSIONS}

Although uterine fibroids represent a very common pathology affecting women worldwide, acute urinary retention due to fibroid compression is a rare event, scarce cases being reported so far. In such cases the option of choice varies according to fibroids' location, size but also according to patients' age and fertility preservation desire. However, in certain cases total hysterectomy with or without bilateral adnexectomy might be the option of choice in order to alleviate the symptoms. Fortunately, once the mechanical compression induced by the fibroids disappears the urinary signs and symptoms seem to rapidly regress.

\section{Acknowledgement}

This work was supported by the project entitled "Multidisciplinary Consortium for Supporting the Research Skills in Diagnosing, Treating and Identifying Predictive Factors of Malignant Gynecologic Disorders", project number PN-III-P1-1.2PCCDI2017-0833.

Conflict of interest: none declared

\section{REFERENCES}

1. Haskal ZJ, Armijo-Medina H. Uterine fibroid embolization for patients with acute urinary retention. J.Vasc. Interv.Radiol. 2008; 19: 1503-5.

2. Arleo EK, Tal MG. Fibroid-induced acute urinary retention: treatment by uterine artery embolization. Int.Urogynecol.J.Pelvic. Floor.Dysfunct. 2008; 19: 161-5.

3. Yang JM, Huang WC. Sonographic findings of acute urinary retention secondary to an impacted pelvic mass. J.Ultrasound Med. 2002; 21: 1165-9.

4. Mahdy A, Faruqui N, Ghoniem G. Objective cure of urinary retention following laparoscopic hysterectomy for a large uterine fibroid. Int.Urogynecol.J. 2010; 21: 609-11.

5. Schwartz Z, Dgani R, Katz Z et al. Urinary retention caused by impaction of leiomyoma in pregnancy. Acta Obstet. Gynecol.Scand. 1986; 65: 525-6.

6. Friedman AJ. Acute urinary retention after gonadotropin-releasing hormone agonist treatment for leiomyomata uteri. Fertil. Steril. 1993; 59: 677-8.

7. Azadzoi KM, Goldstein I, Siroky MB et al. Mechanisms of ischemia-induced cavernosal smooth muscle relaxation impairment in a rabbit model of vasculogenic erectile dysfunction. J.Urol. 1998; 160: 2216-22.

8. Barnacle $S$, Muir T. Intermittent urinary retention secondary to a uterine leiomyoma. Int.Urogynecol.J.Pelvic. Floor.Dysfunct. 2007; 18: 339-41.

9. Barnacle $S$, Muir T. Intermittent urinary retention secondary to a uterine leiomyoma.
Int.Urogynecol.J.Pelvic.Floor.Dysfunct. 2007; 18: 339-41.

10. Andrada AO, De Vicente JM, Cidre MA. Pelvic plexus compression due to a uterine leiomyoma in a woman with acute urinary retention: A new hypothesis. Int. Urogynecol.J. 2014; 25: 429-31.

11. Singh S, Jena SK, Naik M et al. Uterine Fibroid (Leiomyoma) with Acute Urinary Retention: A Case Series. J.Clin.Diagn.Res. 2016; 10: QR01-QR03.

12. Wu CQ, Lefebvre G, Frecker $\mathrm{H}$ et al. Urinary retention and uterine leiomyomas: A case series and systematic review of the literature. Int.Urogynecol.J. 2015; 26 : 1277-84. 